Int. J. Morphol.,

33(1):275-278, 2015

\title{
Nuevo Método de Inclusión en Resina Poliéster P-4 para Cortes Anatómicos
}

\author{
New Method for P-4 Polyester Resin Inclusion for Anatomical Slices
}

Marcos Valenzuela O.*; Bruno Catoia F.*; Aleksandar Munjin P.* \& Fabio Valdés G.*

VALENZUELA, O. M.; CATOIA, F. B.; MUNJIN, P. A. \& VALDÉS, G. F. Nuevo método de inclusión en resina poliéster P-4 para cortes anatómicos. Int. J. Morphol., 33(1):275-278, 2015.

RESUMEN: La resina poliéster (P-4) se ha utilizado en varias técnicas anatómicas, gracias a su bajo costo, fácil manejo y obtención. Entre ellas la inclusión, que se realiza en capas dejando muestras relativamente opacas y de un grosor que no permite ver claramente las estructuras. También encontramos la plastinación de cortes anatómicos, técnica más sofisticada, compleja y de un alto costo económico. El objetivo fue idear un método de inclusión para cortes anatómicos, que mejore la calidad de la técnica clásica. Se utilizaron cortes anatómicos de segmento distal de miembro inferior humano y de un grosor promedio de $0,5 \mathrm{~cm}$ fijados con acetona y cortes de encéfalo de vacuno de igual grosor, fijados con formalina acuosa al 10\%. La totalidad de las muestras son deshidratadas en acetona a $-17^{\circ} \mathrm{C}$. Posteriormente se impregnan e incluyen en resina poliéster (P-4) a temperatura ambiente. Se obtuvieron muestras con una buena transparencia y solidez que permite ver en detalle muchas estructuras, similar a la obtenida con la técnica de plastinación en resina. Mediante un método relativamente fácil de realizar es posible obtener piezas anatómicas de muy buena calidad.

PALABRAS CLAVE: Preservación de tejidos; Inclusión; Resina poliéster.

\section{INTRODUCCIÓN}

La resina poliéster o P-4, gracias a su bajo costo y fácil manipulación, se ha utilizado en múltiples técnicas anatómicas tales como inclusiones, repleciones, corrosiones, diafanizado y plastinación (Von Hagens et al., 1987; Henry $\&$ Latorre, 2007). La plastinación en resina es una técnica que logra preparados anatómicos con muy buena calidad en cuanto a la visualización de estructuras, con buena durabilidad y fácil manejo para fines de docencia en morfología (Valenzuela et al., 2012). Sin embargo, es más compleja y de un costo elevado lo que limita su desarrollo.

Por otro lado, en la técnica clásica de inclusión en resina se incluyen las muestras en capas de dicho material, muchas veces resultando preparados anatómicos opacos y de un grosor que dificulta la visualización de algunas estructuras.

El objetivo de este estudio fue idear un método de inclusión en resina para cortes anatómicos, que mejorara la calidad de la técnica clásica, con resultados similares a la plastinación en resina pero a un menor costo y menor complejidad.

\section{MATERIAL Y MÉTODO}

Se utilizaron muestras anatómicas humanas y de animales divididas en dos grupos (A y B) de acuerdo a su procedencia, con procedimientos similares pero en distintos periodos.

El grupo A se conforma de 10 cortes transversales (tipo medallón) de miembro inferior (pierna) y 5 cortes parasagitales de pies humanos de grosor $0,5 \mathrm{~cm}$ fijados con acetona al $97 \%$ a $-17{ }^{\circ} \mathrm{C}$ durante 20 días.

El grupo B, conformado por 10 cortes coronales y horizontales de encéfalo de vacuno de $0,5 \mathrm{~cm}$, fijados con formalina acuosa al 10\%, durante 20 días. En algunos de estos se tiñó la sustancia gris con el método de Barnard \& Brown (Bravo, 2006) previo a la deshidratación.

La deshidratación de ambos grupos de muestras se realizó con acetona de $97 \%$ y $100 \%$ en forma ascendente a $17^{\circ} \mathrm{C}$ durando 20 días, controlando el grado de deshidratación (Valdés et al., 2010; Valenzuela et al.).

Una vez deshidratadas las muestras fueron retiradas de la acetona y se trasladaron a una cámara frigorífica a $4{ }^{\circ} \mathrm{C}$

* Departamento de Morfología, Facultad de Medicina, Universidad de los Andes, Santiago, Chile. 
donde se mantuvieron por dos horas y posteriormente se cambiaron a una temperatura ambiente de $20^{\circ} \mathrm{C}$ aproximadamente.

Luego, dentro de una cámara ventilada, se preparó un recipiente con resina poliéster sin catalizador ni acelerante donde se dejaron las muestras sumergidas durante 48 horas, cubriendo el recipiente con un paño oscuro. Paralelamente se armaron cámaras de vidrio para incluir los cortes ya impregnados y proceder a la etapa de polimerización con luz ultravioleta (Valenzuela et al.).

Para el armado de las cámaras, se utilizaron dos placas de vidrio de $25 \times 15 \mathrm{~cm}$. Entre estas placas, a unos dos centímetros del margen, se colocó un tubo de silicona con lumen de $5 \mathrm{~mm}$ y grosor completo de $8 \mathrm{~mm}$ de diámetro y $80 \mathrm{~cm}$ de largo que lo recorre en todo su perímetro. Estos vidrios se unen con doble clips (dos a tres por cada lado) dejando solamente uno de los lados de la cámara abierto para introducir la resina y las muestras (Fig. 1). Se rellenó un tercio de ella con resina y se colocaron múltiples muestras en cada cámara de vidrio de tal modo que no se topen entre si. Posteriormente se rellenó con resina hasta cubrir las muestras por completo. Se unieron los tubos de silicona entrecruzándolos, procurando evitar que queden espacios con aire y burbujas al interior (Bravo; Valenzuela et al.). Se colocaron los clips faltantes. Finalmente, el borde de unión de los tubos de silicona se ocluyó con silicona de sellado para evitar que la resina líquida discurra hacia fuera.

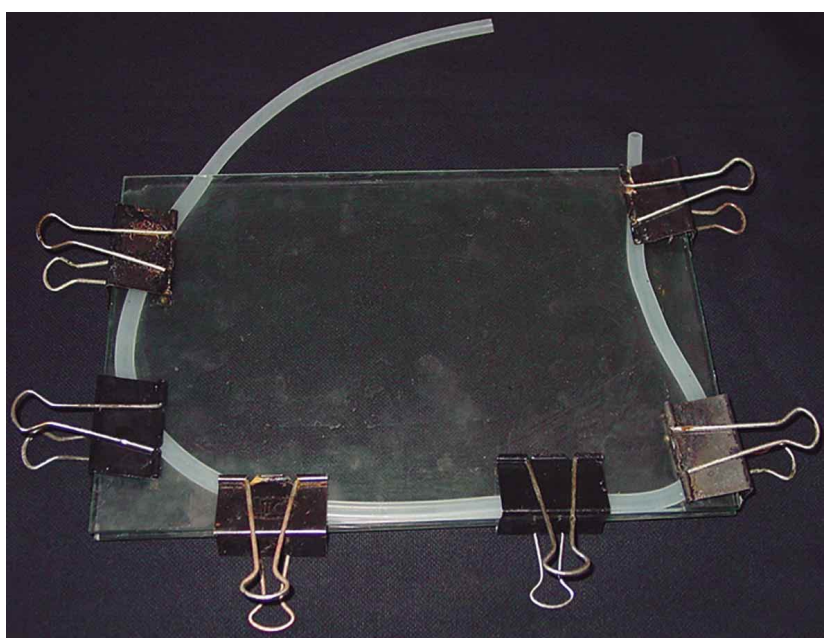

Fig. 1. Las cámaras de vidrio para inclusión se fabricaron con placas de vidrio unidas por doble clips. El espacio para el material a incluirse se obtiene con un tubo de silicona que rodeaba todo el perímetro excepto un lado.

Se dejó reposar colocando la cámara en forma vertical hasta que la silicona haya fraguado totalmente (Fig. 2).

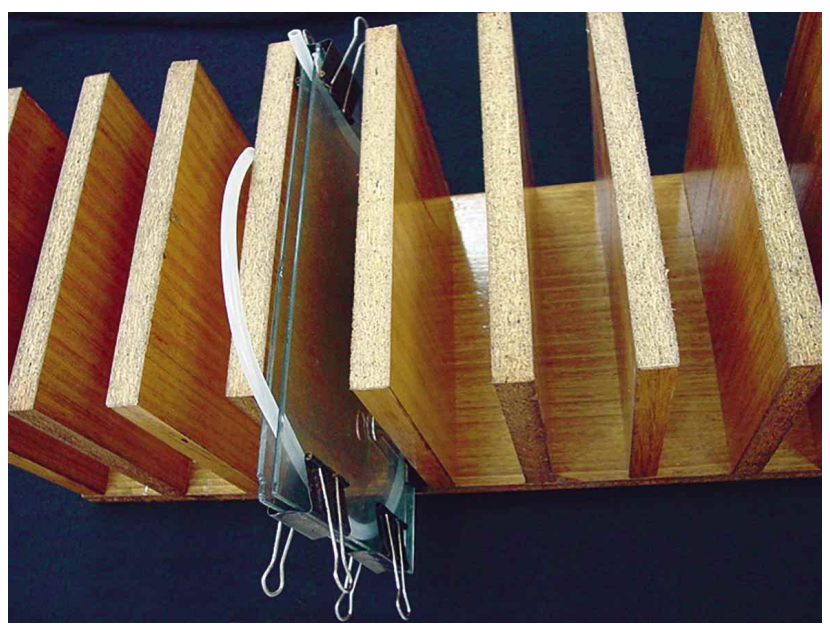

Fig. 2. Soporte confeccionado para dejar reposar las cámaras de vidrio verticalmente hasta el fraguado completo.

A continuación se colocaron las cámaras de vidrio en forma horizontal entre dos focos de luz UV, cubriéndolas con paños oscuros (Fig. 3).

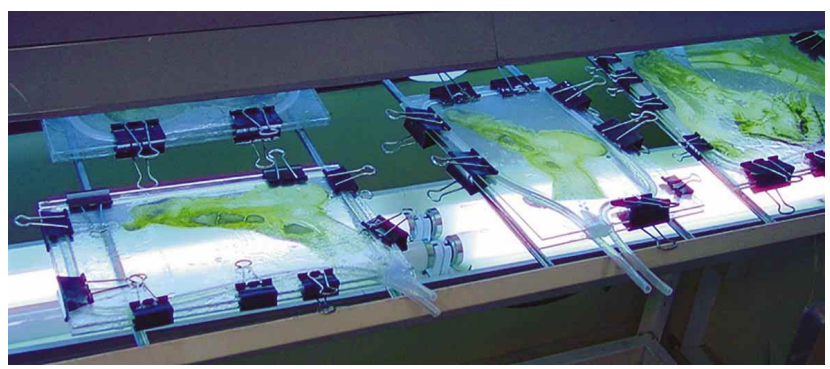

Fig. 3. Cámaras de vidrio en forma horizontal entre dos focos de luz UV.

El proceso de polimerización se efectuó durante el día en un tiempo general de 11 horas con exposiciones continuas de 1 hora a la UV, seguida de intervalos de 30 minutos sin luz. Durante la noche se detuvo el proceso. Las muestras fueron expuestas a la luz UV durante un promedio de 15 horas. Transcurrido este tiempo, se retiraron los clips y el tubo de silicona, se dejó en las cámaras en forma vertical por un par de horas. Luego se desprendieron las muestras de los vidrios, se envolvieron con un film plástico adherente (Fig. 4) y se dejaron sobre una superficie plana cubriéndolas con paños oscuros.

Transcurrida una semana, se procedió a recortar con una sierra la resina sobrante y se guardaron las muestras en cajas plásticas listas para su uso. 


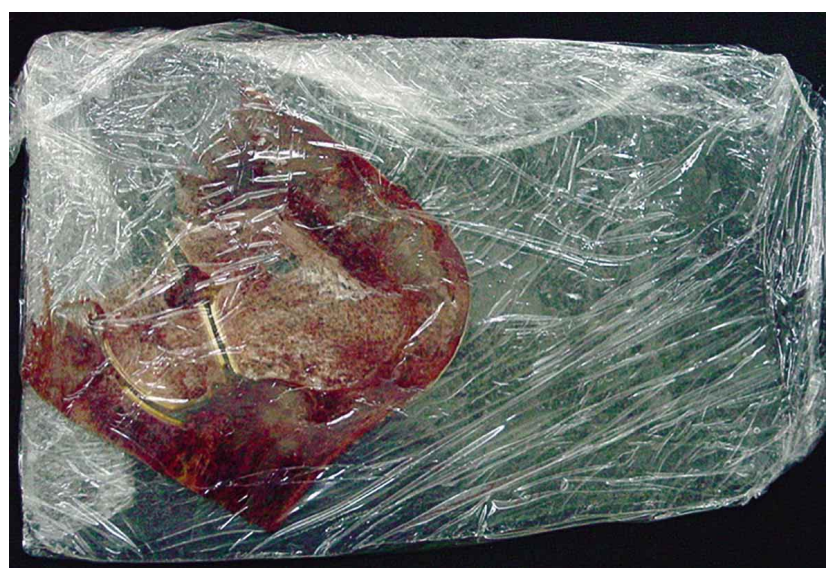

Fig. 4. Muestra ya incluida y cubierta por un film plástico.

\section{RESULTADOS}

Se obtuvieron preparados de cortes anatómicos incluidos en resina poliéster tipo P-4 sólidos y rígidos, de superficie lisa, con una mayor transparencia comparada con la técnica tradicional de inclusión y bastante similar a la lograda en la plastinación con resina (Fig. 5). Además, no se observaron alteraciones en la forma ni tamaño de la muestra.

Algo que preocupaba era la decoloración de los cortes de encéfalo, pero afortunadamente no se ve alterada su coloración.

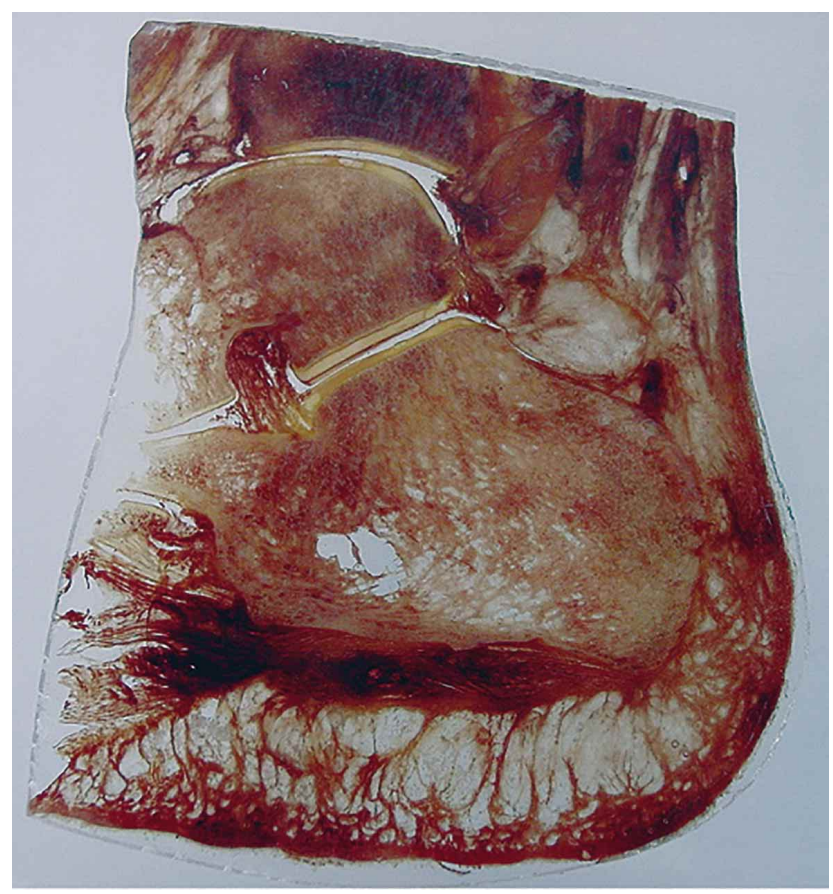

Fig. 5. Cortes anatómicos incluidos en resina poliéster P-4.

\section{DISCUSIÓN}

Durante el proceso de inclusión en resina existen varios detalles que vale la pena discutir. Con el fin de evitar una retracción exagerada de los tejidos por un aumento brusco de temperatura se recomienda cambiar gradualmente las muestras ya deshidratadas a temperatura ambiente pasando por una intermedia a $4{ }^{\circ} \mathrm{C}$ (Valdés et al.; Valenzuela et al.).

Para prevenir un oscurecimiento de las muestras en resina por efecto de la luz natural es aconsejable cubrirlas con paños oscuros desde la etapa de impregnación hasta que esté totalmente polimerizada.

Es bueno que el interior de las cámaras de vidrio tenga un espacio de $2 \mathrm{~mm}$ más alto que el grosor de los cortes, para que estos entren fácilmente en su interior y queden totalmente impregnados, sin embargo no es aconsejable que esta diferencia sea mayor pues las muestras pueden quedar sobre montadas unas sobre otras o la inclusión de un grosor que no permite destacar las estructuras claramente.

Es recomendable colocar un ventilador de aire frio, que funcione en forma paralela a la luz UV, para evitar que las muestras se quemen con el sobrecalentamiento de la resina por efecto de la reacción exotérmica que produce al polimerizar (Latorre \& Henry, 2007).

Al finalizar el proceso de polimerización, el colocar las cámaras de vidrio en forma vertical una vez polimerizada la resina facilita el desprendimiento de las placas de vidrio de la inclusión.

Se aconseja mantener las muestras sobre una superficie plana durante un tiempo (de unos dos meses), hasta que la resina haya polimerizado totalmente, con el fin de evitar el bandeo o encorvamiento de ellas (Latorre \& Henry; Henry \& Latorre).

Con esta nueva técnica, simple de realizar, sin la necesidad de cámaras especiales de impregnación se pueden obtener preparados de cortes anatómicos incluidos en resina de una calidad muy similar a la obtenida con la plastinación con este polímero, con procesos similares, pero con un costo económico considerablemente inferior. Finalmente, se aconseja trabajar la resina en lugares ventilados por su alta toxicidad.

En conclusión, con una técnica de bajo costo, que no requiere de equipos sofisticados tipo cámaras de vacío como en la plastinación, es posible obtener preparados anatómicos de muy buena calidad en cuanto a solidez, durabilidad y 
transparencia haciéndola una alternativa muy viable de reproducir. Esperamos a futuro objetivar estos resultados me- diante un estudio comparativo de las distintas técnicas de inclusión.

VAlenzUela, O. M.; CATOIA, F. B.; MUNJIN, P. A. \& VALDÉS, G. F. New method for P-4 polyester resin inclusion for anatomical slices. Int. J. Morphol., 33(1):275-278, 2015.

SUMMARY: The polyester resin (P-4) has been used in several anatomical techniques, due to its low cost, easy handling and elicit. Among them, the inclusion, which is performed on samples leaving relatively opaque layers and an important thickness which does not allow a clear vision of the structures. There is also the plastination of anatomical slices with this polymer which although, technically sophisticated and complex has a high economic cost. The objective is to develop a method for anatomical section inclusion, improving the quality of classic technique. Distal segment of human lower limb anatomical slices were used with an average thickness of $0.5 \mathrm{~cm}$ fixed with acetone and bovine brain slices equal thickness, fixed with $10 \%$ aqueous formalin. All the samples are dried in acetone at $-17^{\circ} \mathrm{C}$. Subsequently, they are impregnated and included on polyester resin (P-4) at room temperature. Samples were obtained with good transparency and solidity that allows observation of details of many structures, similar to that obtained with the resin plastination technique. Using this relatively easy method we can get very good quality anatomical samples.

KEY WORDS: Tissue preservation; Inclusion; Polyester resin.

\section{REFERENCIAS BIBLIOGRÁFICAS}

Bravo, H. Plastination, an additional tool to teach anatomy. Int. J. Morphol., 24(3):475-80, 2006.

Henry, R. W. \& Latorre, R. Polyester plastination of biological tissue: P40 technique for brain slices. J. Int. Soc. Plastination, 22:59-68, 2007.

Latorre, R. \& Henry, R. W. Polyester plastination of biological tissue: P40 technique for body slices. J. Int. Soc. Plastination, 22:69-77, 2007.

Valdés, F.; Vega, E. \& Valenzuela, M. Two different plastination techniques comparative study. Int. J. Morphol., 28(3):783-6, 2010.

Valenzuela, O. M.; Azocar, S. C.; Werner, F. K.; Vega, P. E. \& Valdés, G. F. Plastination experience in polyester resin (P-4). Int. J. Morphol,. 30(3):810-3, 2012.

Von Hagens, G.; Tiedemann, K. \& Kriz, W. The current potencial of plastination. Anat. Embryol. (Berl.), 175(4):411-21, 1987.

\author{
Dirección para Correspondencia: \\ Dr. Fabio Valdés $G$. \\ Monseñor Álvaro del Portillo 12455 \\ Las Condes, Santiago \\ CHILE
}

Email: favaldesg@uandes.cl

Recibido: 16-10-2014

Aceptado: 19-01-2015 